\title{
Le persuasif et le convaincant : pour une caractérisation fonctionnelle des interventions éducatives en sciences
}

Auteurs

Patrice Potvin, Université du Québec à Montréal, Canada, potvin.patrice@uqam.ca

Marianne Bissonnette, Université du Québec à Montréal, Canada, bissonnette.marianne@uqam.ca

Patrick Charland, Université du Québec à Montréal, Canada, charland.patrick@uqam.ca 


\section{REVUE HYBRIDE DE L'ÉDUCATION}

\section{Résumé}

Adressé aux professionnel.le. s et aux chercheur.e.s, cet article propose une réflexion sur les problèmes et risques associés à certaines utilisations d'étiquettes permettant de qualifier les pratiques et les interventions pédagogiques testées, promues ou contestées dans le cadre de l'enseignement des sciences. Souvent pairées en dichotomies, ces étiquettes sont en effet parfois présentées comme pouvant générer des problèmes de crédibilité des recherches qui les utilisent, ainsi que des incompréhensions et des blessures lorsqu'invoquées imprudemment en formation. II propose ensuite une formule dichotomique et fonctionnelle permettant de caractériser les efforts éducatifs en prenant appui sur la différence ordinaire qui existe dans le langage courant entre la persuasion et la conviction.

Mots-clés: science; enseignement; recherche; formation des enseignant.e.s; persuasif; convaincant 


\section{$\&$}

\section{REVUE HYBRIDE DE L'ÉDUCATION}

\section{Mise en contexte}

\section{Le problème de fonctionnalité des étiquettes}

Bien que pratiquement inévitable, il est toujours brutal, reconnaissons-le, d'attribuer une étiquette unique pour caractériser quelque chose d'aussi complexe et étendu qu'une pratique ou une intervention éducative. Pourtant, cette façon de faire est largement répandue non seulement dans les médias et les journaux, mais aussi dans les milieux scolaires et de la recherche. D'abord, on peut choisir de l'avoir fait par réductionnisme méthodologique. Bien que ce dernier puisse n'entretenir aucune ambition restrictive, il aplatit tout de même la multidimensionnalité du réel pour en tirer une essence unique, utile au chercheur, au moins partiellement injuste. On peut aussi baptiser d'un seul nom une pratique ou une intervention didactique dans le but de pouvoir les présenter de manière abrégée, pour en quelque sorte " faire image ", et ainsi accélérer ou promouvoir quelque démonstration de valeur. II importe cependant de rester lucide quant aux malentendus, aux risques et aux blessures possibles que provoquent souvent de telles réductions.

Dans cet article, nous situerons d'abord dans le monde de la recherche ledit problème de fonctionnalité en présentant certaines des conséquences négatives possibles, typiques des publications et communications issues de la recherche en éducation. Nous présenterons ensuite le problème tel qu'il existe plus clairement encore, mais pour d'autres raisons, dans le contexte de la formation des enseignant.e.s, et $\mathrm{ce}$, en insistant sur la formation continue. Ce n'est que par la suite (en deuxième partie de l'article) que nous proposerons une solution partielle possible aux problèmes qui auront été évoqués en suggérant l'emploi d'une dichotomie que nous croyons possiblement utile. Finalement, nous exposerons une discussion générale sur les ambitions et les limites de la proposition.

\section{Le cas des recherches comparatives}

Une tendance est actuellement observée dans les systèmes scolaires afin que les interventions conduites ainsi que les pratiques employées dans ces systèmes s'appuient davantage sur des connaissances issues de la recherche. Et comme c'est le cas en sciences, les investigations comparatives bien faites sont parmi celles qui présentent les meilleures chances de faire progresser le champ de l'éducation (Geis, 1984). On ne saurait, en effet, être convaincu de la valeur d'une recherche qui se bornerait à présenter les résultats d'une postenquête unique ou alors de simples différences d'effets entre une pré et une postenquête sur telle ou telle variable dépendante à l'étude. Les éventuels progrès enregistrés en seraient inévitablement triviaux et donc, sans grand intérêt, car toute intervention bien intentionnée, même la plus médiocre, fait nécessairement apprendre au moins un peu. On répète alors dans les cours universitaires 


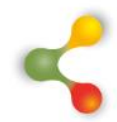

\section{REVUE HYBRIDE DE L'ÉDUCATION}

en méthodologie de la recherche qu'il faut aussi prévoir un groupe témoin, utile non seulement pour sécuriser la comparaison des éventuels gains, mais aussi pour contrôler les effets de test et dans certains cas, d'autres variables confondantes.

Or, dans la recherche en éducation, l'utilisation d'un groupe témoin ou d'une condition contrôle suggère implicitement que les apprenant.e.s qui s'y trouvent ne sont pas soumis à la variable/intervention/pratique testée ou éprouvée, alors que les participant.e.s appartenant à la condition ou au groupe expérimental, eux, le sont. On espère alors obtenir une comparaison de l'effet d'une présence par opposition avec son absence. Tacitement, on assimile la condition " contrôle " à l'eau distillée qu'on utilise souvent en chimie.

Cependant, ce qui se passe dans le groupe témoin n'est jamais nul. De plus, même si on tentait d'y faire vivre le traitement pédagogique le plus banal qui se puisse imaginer, ce dernier impliquerait malgré nos possibles aveuglements volontaires des interactions multiples et synergétiques entre des personnes et le déclenchement de réflexions d'une grande complexité. Le seul discours de l'enseignant.e, si transmissif et unidirectionnel soit-il, reste capable de déclencher des ouragans intellectuels qui répondent bien davantage à la dynamique du chaos qu'à l'édification méthodique. Les itinéraires cognitifs des apprenants, si invisibles soient-ils, peuvent alors réagir de manière imprévisible au moindre mot et déraper vers l'inattendu, ou alors, créer des intelligences magnifiques et extraordinaires, des passions durables comme des blessures profondes. On n'a qu'à songer aux enseignant.e.s qui nous ont le plus inspiré.e.s. Ils ou elles n'ont pas toujours utilisé des techniques d'enseignement sophistiquées; leurs résultats ont toujours largement dépendu de la réceptivité de leurs élèves particuliers et de mille autres variables volatiles.

Nous ne souhaitons pas ici remettre en question la valeur des recherches en éducation sous le prétexte qu'elles s'intéresseraient à des phénomènes trop complexes pour faire l'objet d'un contrôle suffisant. Nous croyons au contraire que de fortes convergences peuvent être enregistrées pourvu que les protocoles de recherche soient bien faits. Ils le sont d'ailleurs tous les jours dans les recherches qui se publient. Nous affirmons cependant que les conditions de contrôles sont inévitablement d'une richesse considérable malgré de possibles suppositions initiales, et que présumer qu'il ne s'y passe rien relève de l'aveuglement.

Donc, pour que les recherches expérimentales ou quasi expérimentales comparatives aient quelque valeur, il est absolument impératif de caractériser le plus précisément possible non seulement ce que contient le traitement expérimental (c.-à-d. la variable indépendante), mais aussi ce que contient le traitement qui sert de base de comparaison (on peut ici choisir de parler de " groupe de comparaison ") ; de dire leurs similitudes et leurs différences. Ainsi, si on enregistre des différences de 


\section{6}

\section{REVUE HYBRIDE DE L'ÉDUCATION}

gains entre les conditions, on saura précisément à quoi imputer cette différence. Bref, sans une caractérisation fonctionnelle et convaincante de chacun des deux groupes comparés (similitudes et différences), au-delà de la présence/absence présumée de la variable à l'étude, la puissance des comparaisons qui les impliquent s'effondre.

Certains chercheur.e.s vont tenter d'esquiver la difficulté. On les voit tester les interventions qu'ils préfèrent, pour de bonnes ou de mauvaises raisons, en les comparant à des traitements qu'ils ou qu'elles qualifieront alors de traditionnels, coutumiers ou habituels. On comprend ici qu'ils ou qu'elles reconnaissent que les groupes contrôles ne sont pas, en définitive, remplis de mannequins et que le discours de l'enseignant.e n'est pas qu'un bruit ou une lecture. Aussi, on soupçonne, souvent à juste titre, que les ambitions de ces chercheur.e.s sont probablement limitées à la formation des enseignant.e.s ; c'est-à-dire qu'ils ou qu'elles sont surtout intéressé.e.s à éventuellement informer les intervenant.e.s et décideur.se.s du monde de l'éducation de ce qui peut se produire dans le cas précis où, par exemple, ils ou elles décident d'implanter tel ou tel mode d'intervention, et ce, par comparaison avec l'éventuel choix de plutôt faire comme d'habitude. C'est une intention fondée sur le but légitime de faire progresser la profession vers de meilleurs dispositifs et solutions.

Reconnaissons aussi que dans plusieurs de ces recherches, les groupes étiquetés de traditionnels ne font pas très souvent l'objet d'observation systématique, ni de caractérisation qui serait suffisamment satisfaisante. Conséquemment, sans disposer d'une connaissance suffisante de ce que contient la base de comparaison utilisée, la valeur nette de l'exercice s'en trouve malheureusement coupée en deux.

II reste évidemment possible que le concept d'enseignement traditionnel et ses synonymes soient fortement évocateurs pour la plupart des personnes impliquées en éducation, et donc, que l'absence de leur description ne soit généralement pas ressentie comme problématique. Cependant, il faut admettre qu'il n'existe aucune garantie que cette évocation soit uniforme d'une personne à l'autre. L'enseignement traditionnel n'est donc pas une variable indépendante qui serait dissociable des cultures institutionnelles ou personnelles dans lesquelles elle serait mesurée ou comprise. On en vient à concevoir qu'il s'agit plutôt d'une sorte d'épouvantail, assez insaisissable et difficilement recevable du point de vue scientifique.

D'autres chercheur.e.s encore, convaincu.e.s de connaître suffisamment ce qui se trouve dans la classe traditionnelle, vont s'autoriser à préciser l'étiquette. La base de comparaison des recherches comparatives qu'ils ou qu'elles mènent s'appelle alors l'enseignement magistral, frontal, direct, voire encyclopédique. L'épouvantail, qu'on présume apôtre du paradigme exclusivement communicatif de l'enseignement, devient alors «bonhomme sept heures». Pratique, on 


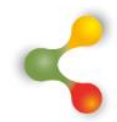

\section{REVUE HYBRIDE DE L'ÉDUCATION}

peut alors beaucoup plus facilement y opposer moralement les méthodes (c.-à-d. les traitements) qu'on présume souhaitables: démarches d'investigation scientifique, approches actives, apprentissage par la découverte, interactions sophistiquées, approches STS, questions socialement vives, changements conceptuels, etc. On se rapproche ici du classique combat du bien contre le mal. Nous nous permettons de vous prévenir : en général, il est prévu que ce soit le bien qui gagne.

II reste cependant bien difficile d'affirmer que les traitements magistraux soient entièrement dépourvus de ce qu'on peut trouver dans la condition expérimentale. Ainsi, on peut très bien imaginer l'existence d'un discours oral qu'un.e enseignant.e adresserait à ses élèves, et portant sur les caractéristiques d'une bonne démarche d'investigation scientifique, les aidant ainsi à mieux réussir ces dernières lorsqu'ils les vivront effectivement. On peut aussi envisager une conférence pertinente qui porterait sur la bonne marche des débats scientifiques, sur les questions socialement vives, ou sur les retombées de la science sur l'économie, l'environnement et la société. Un.e enseignant.e, pour provoquer un changement conceptuel, peut certes faire une démonstration sophistiquée, mais il ou elle peut aussi raconter une anecdote historique bien contextualisée et montrer comment et pourquoi une idée scientifique mérite de triompher sur une autre. En ce sens, étiqueter une intervention pédagogique centrée sur l'enseignant.e ne garantit donc guère qu'elle puisse s'inscrire en opposition totale aux méthodes qu'on juge préférables ou sophistiquées. On peut donc aussi réussir à activer les esprits des élèves même si ceux-ci restent assis et écoutent. Si l'on accepte le moindrement cela, les conclusions issues de comparaisons caricaturées s'en trouvent alors possiblement affaiblies à moins qu'elles n'aient été "pipées » dès le départ par une exacerbation artificielle de la différence entre les groupes, d'ailleurs possiblement préjudiciables aux populations des groupes témoins.

Les métasynthèses et les métanalyses le regrettent elles aussi : les recherches qu'elles recensent et synthétisent expédient trop souvent la caractérisation des groupes contrôles, affaiblissant dans certains cas la valeur des puissants calculs statistiques auxquels elles se livrent pourtant. Dans une métasynthèse portant sur l'intérêt des élèves, Potvin et Hasni (2014) ont par exemple explicitement regretté que les recherches recensées en viennent aussi facilement à des conclusions positives " because of the banality of what happened in the control group " (p. 108). De même, Schroeder et ses collègues (2007), dans la conclusion d'une métanalyse sur les effets de différentes catégories d'interventions en éducation scientifique, se plaignent du fait que les bases de comparaison des traitements dont ils compilent les effets pondérés renvoient à une conception trop floue de l'étiquette " direct teaching " très fréquemment associée à elles. Ils évoquent également les propos de Li et Klahr (2006) qui étendent même leurs doléances jusqu'aux variables indépendantes, c'est-à-dire les étiquettes attribuées aux traitements expérimentaux: 


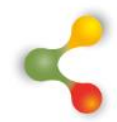

\section{REVUE HYBRIDE DE L'ÉDUCATION}

The "direct instruction" label is sometimes intended to mean a highly-specified instructional procedure, mainly for reading and math, developed by Engelmann et al. (1991): at other times it is used much more diffusely to mean a wide range of teacher-controlled talking, showing, questioning, and demonstrating. "Hands-on science" and "discovery learning" are even less well-defined terms that usually allude to various mixtures of physical manipulation, guided inquiry, and student exploration. Such lack of precise operational definitions has allowed earnest, passionate, but empirically ungrounded debates to flourish in the area of science instruction policy. (p.19)

Les recherches expérimentales et quasi expérimentales comparatives en éducation, qui utilisent des devis expérimentaux et quasi expérimentaux, auraient donc vraisemblablement avantage à mieux surveiller la qualité des étiquettes qu'elles attribuent aux conditions qu'elles contrastent. II y va de la crédibilité de leurs conclusions.

\section{Les discours déployés dans la formation des enseignant.e.s}

D'autres activités en éducation, comme la formation des maitres, auraient également avantage, mais peut-être cette fois pour d'autres raisons, à mieux surveiller leurs caractérisations et leur emploi des " labels ». Durant la tenue de nos activités d'observation de formation des enseignant.e.s, nous avons souvent eu l'occasion de remarquer qu'on utilise bien souvent des étiquettes inconsidérément réductrices. Utilisées trop légèrement ou venant parfois sans explications minimalement satisfaisantes, leur présence devrait immédiatement susciter la suspicion. Toutefois, peut-être en raison de la fréquence de leur utilisation, y sommesnous accoutumés et nos réflexes défensifs semblent alors quelque peu affaiblis?

Dans la formation continue, par exemple, les étiquettes expédiées font des dégâts, mais au contraire des problèmes de crédibilité scientifique qu'ils génèrent en recherche, elles sont ici parfois exploitées par les autorités pour soutenir et alimenter leurs efforts persuasifs. Elles leur sont alors fort utiles. Ne faut-il pas faire progresser l'éducation?

La plus fréquente de ces étiquettes est peut-être celle de l'enseignement-apprentissage centré sur l'enseignant, qu'on oppose alors à celui centré sur l'élève. L'image est puissante, car elle mobilise non seulement une, mais deux étiquettes simultanément et les dichotomise, suggérant une inscription sur un continuum qui va de l'indésirable au souhaitable. 


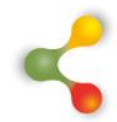

\section{REVUE HYBRIDE DE L'ÉDUCATION}

Avouons qu'il est difficile d'affirmer qu'il n'y a pas quelque chose de vrai et de pertinent dans cette «double étiquette » : on connait tous des enseignant.e.s qui semblent manquer cruellement d'empathie cognitive et de sensibilité aux particularités et aux besoins individuels des personnes à qui ils ou elles enseignent. À l'autre bout du spectre, l'image d'autres enseignant.e.s, bien différent.e.s, nous vient peut-être à l'esprit. Ce sont des personnes qui nous ont interpellés, davantage qu'elles nous ont donnés; qui se sont adressés à nous, et qui nous ont fait vivre des choses, qui nous ont fait grandir, plus qu'elles nous ont simplement parlé de ces choses ou nous informer sur elles. Ce sont de bon.ne.s enseignant.e.s qui ont réussi à nous atteindre et donc, à nous changer; qui veulent faire des "têtes bien faites " plutôt que simplement des "têtes bien pleines" (Fourez, 2002).

Par l'évocation de tels extrêmes, on souhaite peut-être ainsi, dans un élan constructiviste, rappeler que l'intelligence est une force vive, un moulin à construire du sens, une infatigable machine interprétative et que les apprenants ne sont pas tous égaux. On veut rappeler que c'est en l'élève, et par lui, que l'apprentissage se fait, en définitive; et que lui transmettre le savoir par des présentations orales, si parfaites soient-elles, ne peut vraisemblablement pas suffire pour produire des apprentissages et des développements valables ou profonds, ou sécuriser le développement intégral des personnes (Legendre, 1983) et de véritables êtres éduqués (Legendre, 1995).

S'ils peuvent effectivement motiver et même mobiliser certain.e.s enseignant.e.s, les idées de l'enseignement-apprentissage centré sur l'enseignement ou sur l'élève et leurs différentes déclinaisons (paradigme de l'enseignement versus paradigme de l'apprentissage) ne sont malheureusement pas aptes à outiller qui que ce soit. Bien que leurs extrêmes fassent image et supposent la destination, ils ne fournissent entre eux deux rien d'utilisable, ni critère, ni piste de redressement. De plus, bien qu'ils réfèrent à des choses que l'on peut aisément imaginer, dans la réalité, des pratiques qui seraient purement ou suffisamment l'une ou l'autre n'existent tout simplement pas de manière intégrale ou sont impossibles à qualifier absolument.

Puisqu'il se trouve dans chaque situation d'apprentissage à la fois l'enseignant.e et l'élève, et que la présence et la participation des deux est indispensable à la réussite du projet éducatif, on voit mal comment on pourrait choisir d'en exclure un. C'est pourtant ce que fait la dichotomie présentée plus haut. Or, il faut à la fois une plus forte étincelle et un meilleur carburant pour améliorer les performances d'un moteur. Pas l'un au détriment de l'autre.

II n'est pas rare que plusieurs enseignant.e.s, qui pourtant au départ font preuve d'une grande motivation intrinsèque, sortent frustré.e.s des formations continues qui leur sont imposées, et qu'ils ou qu'elles 


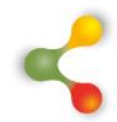

\section{REVUE HYBRIDE DE L'ÉDUCATION}

deviennent ensuite très réfractaires et réticent.e.s à en recevoir d'autres. Nous croyons que cette réaction représente le résultat non pas tant de la piètre qualité des contenus pédagogiques qui leur auront été proposés, mais de la manière dont on a qualifié ces contenus et dont on a qualifié leurs contraires supposés.

Par exemple, on n'hésite pas à présenter certaines interventions éducatives pourtant largement utilisées dans les écoles comme étant insuffisamment centrées sur l'élève. Cependant, un tel jugement peut être très difficile à accepter pour certains enseignant.e.s, qui sont parfois contraint.e.s de recourir à de telles interventions, non pas parce qu'ils ou qu'elles les auront choisies, mais par la surcharge qui leur est imposée (grands groupes, horaires soutenus, etc.).

Ils ou elles peuvent alors avoir l'impression légitime qu'on qualifie certains éléments de leurs préférences pédagogiques comme manquants d'altruismes. Compte tenu de la quantité considérable d'énergie que les enseignant.e.s déploient pour accomplir et optimiser les retombées de leur travail auprès des élèves, il est absolument normal, regrettable, mais prévisible, que certains reçoivent tout cela comme une gifle. Certains enseignant.e.s vivent littéralement pour leurs élèves. Ils ou elles planifient l'année scolaire prochaine durant leurs vacances, sont constamment attentif.ve.s à tout ce qui pourrait les aider à remplir leur rôle social de faire réussir. Convaincu.e.s de l'importance de leur action sur des personnes en développement qui sont souvent vulnérables, certain.e.s pousse.nt leur engagement jusqu'à l'épuisement. Nous ne croyons donc pas moralement acceptable de leur souffler que leur pratique n'est pas suffisamment centrée sur l'élève et d'ainsi, volontairement ou non, remettre en question la qualité des motifs fondamentaux de leur implication.

Encourageant pour certains, accablant pour d'autres, l'usage peu avisé et maladroit d'étiquettes pouvant être perçues comme culpabilisantes aura aussi pour effet d'augmenter la fracture du personnel enseignant en deux groupements absolument stériles, alors que la solidarité dans l'adversité devrait au moins être une part de la solution : les innovants et les résistants.

II existe aussi d'autres tentatives semblablement maladroites pour caractériser les interventions utilisées en classe. L'apprentissage actif versus l'apprentissage passif en est une. Reconnaissons d'abord que la recherche montre avec une clarté certaine que l'apprentissage est plus facilement issu de l'activité que de la passivité. Certaines pratiques d'enseignement qui la contraignent ou l'encouragent, sont effectivement meilleures : la prise de position volontaire et consciente (Albe, 2005) ; la formulation explicite d'hypothèses ou de prédictions (Brod, Hasselhorn et Bunge, 2018); l'obligation de contribuer aux débats (Lilly, 2012) ; la possibilité de faire des choix (Hasni et Potvin, 2015); la réflexion métacognitive (Schraw, Crippen et Hartley, 2006) ; la contrainte d'une 


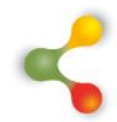

\section{REVUE HYBRIDE DE L'ÉDUCATION}

intention de lecture (Boudreau et Beaudoin, 2015) ; la prise en charge de la résolution des problèmes (Reigosa et Jiménez-Aleixandre, 2007) ; etc.

Cependant, voilà que la plupart du temps, on présume que certaines interventions sont en elles-mêmes des pratiques qui, intégralement ou essentiellement, entretiennent la passivité ou l'activité, alors qu'en réalité, l'analyse de ces pratiques en leurs morceaux révèle de multiples qualifications possibles. On peut aussi, par exemple, donner des cours "magistraux ", présumément platoniques, mais qui déclenchent de véritables tempêtes idéologiques, alors que les élèves paraissent assis «bien sagement » sur leur chaise. On peut aussi faire visionner un film bien choisi qui présente le potentiel de déclencher de violents conflits cognitifs et même, possiblement, des changements conceptuels, initialement souhaités. À l'inverse, on peut aussi organiser une belle sortie scolaire présumément active, debout, dynamique, mais en définitive, cognitivement stérile. On peut faire vivre une expérience de laboratoire exigeante qui ne soit finalement que platement vérificative, tout comme on peut obliger un débat " scientifique » animé, mais qui ne tourne finalement qu'en conflit d'opinions. On peut faire jouer à un jeu vidéo éducatif fort engageant, mais sans que les gains souhaités ne soient au rendez-vous.

Ainsi, il n'y a pas que les grandes méthodes pédagogiques déployées qui comptent. II y a aussi les adhésions épistémologiques et les croyances pédagogiques des enseignant.e.s, ainsi que tous les petits gestes, les petits mots, les contraintes subtiles imposées, les relances et les petits effets qui découlent de ces adhésions et qui font la différence. Ces derniers s'avèrent même, dans certains cas, encore plus déterminants que la grosse quincaillerie d'origine idéologique utilement déployée et éventuellement célébrée.

D'autres dichotomies sont quant à elles utilisées en formation continue dans le but de marquer la direction souhaitée (situation actuelle $\rightarrow$ situation projetée), mais sans qu'il n'y ait d'opposition absolue entre les étiquettes adverses. Ainsi, parfois, on oppose maladroitement et sans développement suffisant le transmissif et le constructiviste, laissant ainsi croire que la construction des savoirs est souhaitable et même possible, sans un minimum de transmission d'information ou de base conceptuelle suggérée. On oppose de la même manière l'enseignement par accumulation de savoirs avec la pédagogie par compétences, en laissant penser que les compétences se développent indépendamment de l'existence des savoirs. On contraste la répréhensible répétition (ou le par cœur) avec la souhaitable compréhension, alors que tous les musiciens et athlètes compétents vous jureront qu'ils excellent par la pratique répétée. On contraste aussi l'autoritaire et le démocratique, sans rappeler que tout dans la classe ne peut être obtenu par délibération et qu'il faut malgré tout un chef « qui sait mieux » et sans lequel il ne peut y avoir de destinations, de discipline ou de progrès suffisamment rapide. Les élèves doivent aussi reconnaître suffisamment d'autorité intellectuelle à ou de confiance en 


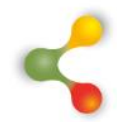

\section{REVUE HYBRIDE DE L'ÉDUCATION}

l'enseignant.e pour ne pas douter constamment de la valeur de ce qu'il ou qu'elle affirme. Tout cela n'empêcherait en rien un.e enseignant.e qui serait ainsi autoritaire de faire vivre et ressentir à ses élèves les dimensions démocratiques du développement des savoirs scientifiques.

Finalement, en enseignement des sciences en particulier, on oppose parfois les processus et les produits, ou l'apprentissage par approche communicative avec l'apprentissage par la découverte.

L'emploi de ces deux dichotomies encourage la réalisation d'agendas à peine cachés. Ainsi, s'intéresser aux processus de résolution est présenté comme plus intéressant que d'évaluer les produits, car les processus mènent justement à ces derniers. Ne s'intéresser qu'à la valeur des réponses, par exemple, nous enlèverait le moyen de les améliorer. Mais la qualité des productions et des réponses n'est-elle tout de même pas une indication valable, bien qu'insuffisante, à l'effet que les processus ont été bien menés ? Dans ce contexte, on peut se demander pourquoi ne pas considérer les deux plutôt que de les opposer et alors risquer le malentendu?

De même, il n'est absolument pas exclu qu'il soit possible et profitable de faire découvrir aux élèves certaines observations, relations, et même quelques lois scientifiques par des expérimentations ou des démonstrations cruciales, des démarches exploratoires, ou des débats bien organisés, mais voilà : de tels dispositifs pédagogiques d'apprentissage par la découverte sont, lorsqu'ils fonctionnent, pilotés très étroitement par des enseignant.e.s astucieux.ses qui savent exactement où ils amènent leurs élèves (Kirschner, Sweller et Clark, 2006). Ils ou elles les guident alors rigoureusement avec des cahiers des charges ingénieux et bien construits dans lesquels ils ou elles auront su leur communiquer toutes les informations nécessaires pour les faire passer par le diabolique entonnoir préprogrammé et assurer qu'ils soient suffisamment outillés pour prendre en charge par eux-mêmes la résolution. On est donc contraints de reconnaître que dans l'acte d'enseigner par la découverte, il y a parfois beaucoup d'informations, mais surtout les bonnes. Pas nécessairement toutes les informations, mais précisément celles qui générèrent les prises de conscience et les découvertes souhaitées. Cessons donc d'opposer communication et découverte; il y a de l'une dans l'autre pour tout projet éducatif qui sait où il s'en va. De surcroît, admettons qu'il faudrait être extraordinairement naïf pour espérer l'apprentissage par la découverte au point de croire qu'on peut faire reconstruire morceau par morceau, par chacun des élèves, l'ensemble des connaissances scientifiques bien présentes dans le programme, mais qui ont mis des siècles à être développés par des communautés entières de scientifiques professionnels. Reconnaissons aussi qu'on ne peut pas toujours douter de tout et qu'à partir d'un certain moment, une certaine confiance en l'institution scientifique finit par être souhaitable pour favoriser un développement accéléré de la culture. Ainsi, communication et découverte sont tous deux 


\section{6}

\section{REVUE HYBRIDE DE L'ÉDUCATION}

indispensables et participent à l'essor culturel. Les présenter comme opposés laisse croire qu'il faut obtenir l'un au détriment de l'autre, ce qui est non souhaitable, et ce, bien que nous avons souvent vu des enseignant.e.s croire qu'apprendre par la découverte signifie laisser faire total, pour évidemment échouer et ensuite renoncer complètement à faire autre chose que donner des informations.

Or, les enseignant.e.s ne sont généralement pas dupes des dichotomies stérilement présentées, mais ils ou elles ne comprennent pas pourquoi on les leur sert. Dans les formations continues, entre autres, on ressent beaucoup de résistance qui pourrait s'avérer justifiée. Peut-être ne la ressent-on pas autant dans les contextes de formation initiale où les futur.e.s enseignant.e.s n'ont peut-être pas encore eu l'occasion d'éprouver la valeur utilitaire de telles étiquettes binomiales ? Le choc sera quand même là, seulement différé.

Chercheur.e.s, formateur.rice.s d'enseignant.e.s, il est de notre devoir moral d'attribuer l'étiquette qui représente le mieux possible l'objet qu'elle tente de dépeindre, en tout respect de la réalité professionnelle, et même si on doit quand même motiver les troupes et les mobiliser.

\section{Développement}

\section{Le persuasif et le convaincant : un positionnement didactique}

Nous ne souhaitons pas ici résister à l'emploi des dichotomies. Ces dernières sont puissantes et permettent parfois d'expliquer efficacement. Cependant, selon nous, retenir des extrêmes trop éloignés l'un de l'autre laisse exister un trop grand nombre de cas intermédiaires et laisse alors sévir les malentendus et les confusions quant aux efforts à faire.

Nous souhaitons donc présenter ici une dichotomie que nous n'avons pas encore vue apparaître sous cette forme particulière dans la littérature de recherche en éducation scientifique et qu'il est possible d'envisager pour baliser certains efforts éducatifs en recherche ou en formation. Cette dichotomie ne parviendra certainement pas à recouvrir l'ensemble des possibles de manière utile, ni à remplacer toutes les dichotomies présentées précédemment, car telle n'est pas sa fonction présumée. Nous croyons cependant qu'elle pourrait contribuer, dans certains cas, à apaiser certains problèmes évoqués plus haut. C'est donc une proposition à intention bienveillante que nous soumettons à la communauté éducative, en sciences en particulier.

Basée sur la nature des contenus particuliers qui caractérise l'activité scientifique (et peut-être des technologies), la proposition est donc de nature davantage didactique que pédagogique. Elle pourrait donc s'avérer plus fonctionnelle et utile que plusieurs autres dichotomies dans les cas où l'on tenterait d'étiqueter ou de caractériser des traitements éducatifs qui 


\section{$\&$}

\section{REVUE HYBRIDE DE L'ÉDUCATION}

souhaiteraient augmenter leur sensibilité aux propriétés particulières des savoirs et des méthodes scientifiques.

Cette dichotomie est basée sur la différence ordinaire qu'on retrouve dans le langage courant (dictionnaires ordinaires et autres) entre la persuasion et la conviction. Déjà, certains lecteur.rice.s pourraient douter qu'il puisse exister une différence instructive ou significative entre les deux. Cette réaction est très positive, car non seulement elle entraîne la curiosité, mais de plus, la grande parenté qui existe entre eux autorise aussi une évacuation du débat de plusieurs tensions qui n'ont pas lieu d'être.

En effet, dans les deux cas, des enseignant.e.s (agent.e) et leurs élèves (sujets) sont présent.e.s et impliqué.e.s. Dans les deux cas, des informations pertinentes, utiles et même essentielles peuvent être apportées par voie orale et magistrale. Dans les deux cas, on présume de l'activité des apprenant.e.s, de leur travail soutenu, de leur bonne foi et de leur capacité à s'adapter aux circonstances (milieux). De même, on accepte sous chaque étiquette que le bachotage, le " teach to the test » ou l'enseignement par raccourci conceptuel, sont à proscrire. Dans les deux cas, on reconnaît que le respect des programmes d'étude (objet) fait partie du travail de l'enseignant.e, qu'il est possible d'étiqueter de grandes méthodes comme de tout petits gestes, qu'il est possible que l'enseignant.e doive recourir à des interventions de l'un ou de l'autre " type » pour des raisons qui sont hors de son contrôle.

La dichotomie donne aussi du grain à moudre : parfois, pour une même quantité d'énergie déployée, et toutes autres choses étant égales par ailleurs, il reste possible d'enseigner différemment, pourvu que les options soient connues.

\section{L'enseignement persuasif}

Dans le contexte de l'éducation aux sciences, l'enseignement persuasif pourrait être défini comme tout enseignement qui cherche à obtenir efficacement l'adhésion de l'apprenant.e aux propositions scientifiques programmées.

On ne cherche pas ici à réduire l'idée de proposition scientifique à des énoncés écrits ou oraux, ou à leur formulation, mais l'étendre à toute connaissance qui présente quelques utilités dans l'activité scientifique. On pourrait alors l'élargir non seulement à celles qui, discursives, seraient le produit de l'activité scientifique (concepts, lois, principes, etc.), mais aussi à toute proposition de nature méthodologique ou de compétences scientifiques. On peut même penser aux attitudes et aux valeurs, pourvu que celles-ci soient issues, comme les autres objets considérés, des programmes de formation en sciences. 


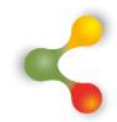

\section{REVUE HYBRIDE DE L'ÉDUCATION}

Ceci étant dit, le motif principal et classique dans l'enseignement persuasif reste l'obtention d'adhésions, c'est-à-dire l'exigence ultime d'obtenir que l'apprenant.e fasse sien.ne de telles propositions, qu'il.le y souscrive ou en reconnaisse la valeur absolue, qu'il.le les approuve. De telles adhésions peuvent recouvrir au moins partiellement les idées de compréhension, de mobilisation, d'utilisation et de réflexion relativement aux propositions concernées. On peut en effet légitimement chercher à obtenir par la persuasion que les élèves acceptent, mais aussi comprennent, mobilisent et réfléchissent les propositions d'ordre scientifique.

L'adhésion s'obtient par des moyens couramment utilisés aujourd'hui dans nos écoles. Ainsi, des enseignant.e.s persuasif.ve.s s'emploient à démontrer aux élèves la valeur descriptive, explicative, prédictive et exploitationelle (Potvin, 2018) des propositions programmées, et ce, par la présentation de cas qui sont essentiellement positifs. En d'autres termes, ces enseignant.e.s vont fournir des preuves de toutes sortes (expérimentales, autoritaires, expérientielles, observationnelles, démonstratives, des exemples, etc.), susceptibles de soutenir ou de renforcer une démonstration de puissance et de rehausser leur valeur, leur utilité ou leur mérite perçus. Bref, on fournit les meilleures preuves possibles qui appuient le mieux la valeur des propositions programmées. C'est à travers ces preuves et ces arguments, leur nombre et leur qualité, qu'on favorise l'adhésion et l'habituation pour ces enseignant.e.s.

Puisque dans ce contexte, c'est surtout le résultat (les « extrants») qui compte plutôt que le moyen d'y parvenir, on pose évidemment le problème de l'efficacité de ces initiatives. On cherche alors à obtenir, le plus vite et efficacement possible, et pour le plus grand nombre d'élèves, les adhésions souhaitées. On les souhaite fortes, aussi. Le temps de classe et l'énergie étant limités, les progrès souhaités immenses, le travail d'enseignement ne se conçoit alors plus autant comme pouvant être complètement réussi, mais plutôt comme pouvant être optimisé.

Les initiatives persuasives sont souvent faciles à reconnaître. Le tableau 1 en présente quelques-unes et indique en quoi elles permettent de favoriser les adhésions.

Tableau 1 : Caractérisation des interventions persuasives

\begin{tabular}{cc}
\hline Intervention & Buts visés \\
\hline $\begin{array}{c}\text { Présentations orales (menées par } \\
\text { l'enseignant.e) exhaustives et bien } \\
\text { construites }\end{array}$ & $\begin{array}{c}\text { Favoriser l'intelligibilité, la clarté, la } \\
\text { plausibilité et la fertilité du propos. }\end{array}$
\end{tabular}




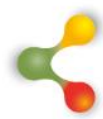

\section{REVUE HYBRIDE DE L'ÉDUCATION}

\begin{abstract}
L'enseignant.e est souvent à l'avant, bien visible par tous les élèves. Ces derniers écoutent attentivement et en silence. Variation des stimuli.

Favoriser l'attention et éviter qu'aucune information cruciale ne soit perdue.
\end{abstract}

Tous les contenus des programmes se voient attribuer une attention similaire et sont considérés importants.

L'enseignant.e doit se rendre au bout de sa planification.

Permettre de favoriser l'acquisition de toutes les cibles.

\section{Réalisation de séries exhaustives d'exercices qui permettent de mobiliser les connaissances}

Apprécier à quel point les connaissances permettent de résoudre des problèmes nombreux et variés, bien qu'apparentés.

Les routines sont favorisées. On met en valeur une seule manière de résoudre les problèmes. Sous pression, on réclame des « actes de foi ».

Expérimentations de laboratoire vérificatives

Fournir une démonstration d'utilité, de puissance ou de vérité de ce qui aura été enseigné auparavant.

Anecdotes historiques et récits « savoureux » qui relatent et valorisent les contributions extraordinaires des chercheur.e.s du passé.
Insister sur les effets bénéfiques des découvertes relatées sur la société, l'environnement, l'économie, etc.

Dans les classes persuasives, on est aussi très attentif à tout ce qui pourrait être gaspillage. Ainsi, l'apprentissage est surtout considéré comme une affaire individuelle, car c'est l'adhésion de chacun qui est en définitive recherchée. Consensus et discussions sont peut-être des concepts à haute désirabilité sociale, mais ils sont aussi parfois considérés comme devant être réalisés avec prudence; si les échanges collaboratifs peuvent favoriser certaines adhésions souhaitables, il arrive aussi que ces échanges les fassent dérailler par contamination. Ainsi, dans ce contexte, le travail d'équipe est surtout utilisé pour des raisons d'économie, ce qui libère du temps pour fournir davantage de preuves positives. Dans l'enseignement persuasif, on hésite à s'intéresser aux erreurs et aux conceptions erronées des élèves. Répétées ou rendues publiques, elles peuvent en effet menacer ou retarder certaines compréhensions importantes. Elles sont parfois considérées comme un bruit indésirable et, règle de base de plusieurs enseignant.e.s d'expérience: " on ne laisse jamais quelque chose de faux écrit au tableau bien longtemps ». On corrige aussi les copies des élèves selon le même esprit : on marque au crayon rouge la faute sur le cahier et on espère que l'élève voie mieux ses manquements aux adhésions recherchées et se mette rapidement en projet de se corriger lui-même. Dans un souci d'efficacité, un enseignement persuasif fait en général l'impasse sur les considérations 


\section{$\&$}

\section{REVUE HYBRIDE DE L'ÉDUCATION}

épistémologiques, psychologiques et métacognitives, car même si on en reconnaît aisément leur valeur absolue, leur apport relatif en salle de classe est plus que douteux considérant que les programmes sont chargés et que les examens ne vont pas là. Dans l'enseignement persuasif, on regrette aussi de ne pas pouvoir être assez attentifs aux besoins des personnes. C'est que le peu de temps dont on dispose nous l'interdit, bien qu'en réalité, plus souvent on croit savoir d'avance ce qu'il leur faut, on le leur donne. On ne s'attarde pas beaucoup non plus à la valeur ajoutée des objets qu'on enseigne. On la présume, et par la suite, on montre ses avantages, sa puissance et l'intérêt qu'elle devrait susciter.

Nous ne souhaitons pas ici condamner cette façon de faire, qui est parfois, c'est-à-dire trop souvent, la seule qui soit viable. Avec des classes surpeuplées et des programmes surchargés, sous la contrainte parfois d'un difficile examen du ministère et la pression des parents, il est normal que des enseignant.e.s se mettent à la recherche, le plus efficacement possible, d'adhésions, tout comme il est normal que compte tenu des difficiles circonstances dans lesquelles on les enferme, ils ou elles finissent par percevoir que tel est le contrat social qu'ils ou qu'elles ont signé avec l'état. Impossible de leur jeter la pierre alors que l'on compte tant sur eux pour tout. II faut plutôt admettre sereinement qu'il est vraisemblablement inévitable qu'une bonne part des interventions éducatives prévues ne puisse être que persuasive, et nous croyons que les très bons enseignant.e.s, lorsque les circonstances l'exigent, savent l'être aussi.

II faut aussi admettre qu'une analyse même superficielle de l'enseignement persuasif nous conduit à y trouver de troublantes ressemblances avec les discours commercial, politique et religieux, qui eux aussi, à coups d'une accumulation de messages, de preuves et d'arguments toujours positifs qui appuient, séduisent, martèlent, et parfois jouent de menaces et utilisent la peur, cherchent à produire l'adhésion. Comme pour la vente, la soumission idéologique et la foi, l'enseignement peut être dogmatique.

De cette façon, il ne faut pas s'étonner, dans les circonstances, que les élèves éprouvent des difficultés à percevoir la différence entre le discours scientifique et le discours pseudoscientifique. Comment les différencier en effet si ces derniers acquièrent leur crédibilité semblablement?

L'enseignement persuasif rencontre aussi une autre difficulté : celle des adhésions concurrentes. Si un.e enseignant.e perçoit à un moment donné que son travail consiste à faire adhérer ses élèves orthodoxes - de quelque confession que ce soit - à la théorie de l'évolution par voie de sélection naturelle, comme peut-il ou elle alors simultanément réussir son projet d'enseigner et assurer le respect des croyances? Comment peut-il ou elle éviter le problème moral qui en découle ? Comment concilier 


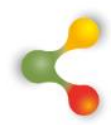

\section{REVUE HYBRIDE DE L'ÉDUCATION}

l'ambition d'éduquer et l'aspect « rouleau compresseur » des initiatives qui visent l'adhésion?

La croyance en la supériorité de la science ne peut cependant pas suffire pour justifier la décision de l'enseigner. Cependant, si l'on choisit de fonder notre réflexion sur sa nature et ses propriétés, cela permettra peutêtre de voir en quoi elle se distingue des autres systèmes de pensée disponibles et donc, en quoi elle constitue une corde authentiquement supplémentaire à l'arc intellectuel.

\section{L'enseignement convaincant}

La science est le contraire du dogmatisme. Seule entreprise à se corriger elle-même, son épistémologie semble lui reconnaître un caractère masochiste. Paradoxalement, c'est cette attitude qui lui confère robustesse, crédibilité, évolution et actualisation. Ce qui résiste le mieux aux assauts « autodestructeurs » serait alors conséquemment ce qui est "le plus vrai » (Popper, 1995). Parmi les auteur.e.s des citations qui reconnaissent peut-être le mieux ce caractère distinctif de l'activité scientifique par rapport aux autres types d'activités, on reconnaît les plus grand.e.s :

Décider de rejeter un paradigme est toujours simultanément décider d'en accepter un autre, et le jugement qui aboutit à cette décision implique une comparaison des deux paradigmes par rapport à la nature et aussi de l'un par rapport à l'autre. (Kuhn, 1962, p. 115)

Une théorie qui n'est réfutable par aucun événement qui se puisse concevoir est dépourvue de caractère scientifique. Pour les théories, l'irréfutabilité n'est pas (comme on l'imagine souvent) vertu, mais défaut. (Popper, De Launay et De Launay, 1985, p. 64)

We are trying to prove ourselves wrong as quickly as possible, because only in that way can we find progress. (Feynman, Cox et Ma, 2015, p. 67)

C'est au moment où un concept change de sens qu'il a le plus de sens. C'est alors qu'il est en toute vérité un événement de la conceptualisation. (Bachelard, 1963, p. 56)

Ainsi, dans ce contexte, adhérer « scientifiquement » ne saurait être une simple affaire d'adhésion volontaire ou enthousiaste, si constructive soit-elle. Ce serait plutôt comprendre pourquoi une proposition dite scientifique serait ou ne serait pas préférable à une autre proposition concurrente, ou alors pourquoi elle aurait quelque valeur par rapport à une norme ou un critère qui soient plus grands que nous-mêmes. Adhérer à la 


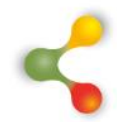

\section{REVUE HYBRIDE DE L'ÉDUCATION}

manière scientifique, ce serait aussi savoir, pour l'avoir vécu, pourquoi telle méthodologie, telle technique, ou telle instrumentation sont avantageuses par comparaison avec d'autres disponibles. En d'autres termes, on doit tenter d'établir la valeur des propositions scientifiques non pas sur la base des seuls avis personnels et absolus, ni même par accumulation de démonstrations de puissance, mais obligatoirement en rapport avec un objet extérieur aux sujets délibérants. Donc, il apparait obligatoire de savoir en quoi telle ou telle proposition revêtirait quelque valeur relative. Un.e enseignant.e contribue à établir cette valeur lorsqu'il ou elle présente les mérites et limites respectifs non seulement du modèle de Darwin, mais aussi de celui de Lamarck ; lorsqu'il ou elle discute avec ses élèves des raisons pour lesquelles les idées spontanées sont toutes initialement recevables pour ensuite les contraster entre elles et avec les modèles à faire apprendre ; lorsqu'il ou elle discute du critère de simplicité (ou de falsifiabilité ou de reproductibilité) de certaines propositions scientifiques ; lorsque les biais cognitifs sont évoqués en classe pour expliquer les résistances et les adhésions ; lorsqu'il tolère les erreurs et en fait un objet pédagogiquement intéressant, etc.

Cette façon de faire intéressera d'ailleurs l'enseignant.e qui se heurte aux croyances religieuses de ses élèves : il ou elle n'est plus tenu.e de faire triompher les propositions scientifiques programmées contre toutes les autres adhésions. II ou elle peut se contenter de montrer leur valeur fonctionnelle ou épistémologique, ou de contraster les pouvoirs explicatif, prédictif ou exploitationnel des propositions à faire apprendre avec d'autres propositions que celles religieuses. II ou elle peut aussi explicitement attribuer la crédibilité de certaines propositions scientifiques à autre chose que son action ou ses adhésions personnelles, comme au travail de crédibilisation des idées qui est effectué par la communauté scientifique.

Évidemment, les seules entités disponibles pouvant reconnaître et accorder de la valeur aux propositions sont les personnes qui les étudient, c'est-à-dire les apprenant.e.s. On rejoint ici le caractère subjectif de l'activité scientifique, mais lorsque ces personnes discutent ensemble du lien qui peut exister entre les idées et les objets ou les événements, c'està-dire les faits, on peut penser qu'on dispose là de la meilleure approximation objective possible (c.-à-d. une communauté délibérante scientifique). Or dans cette dernière, une activité privilégiée est la publication, le débat ou le congrès, dans lesquels les scientifiques se rencontrent et tentent de se convaincre mutuellement de la valeur de leurs propositions respectives au regard desdits faits. Tenter de convaincre apparaît alors comme une entreprise inévitable et profondément scientifique et c'est pourquoi nous tentons d'adapter cette idée à l'enseignement.

Dans le contexte de l'éducation aux sciences, l'enseignement convaincant pourrait alors être conçu comme tout enseignement qui cherche à fournir les informations et les expériences propres à amener 


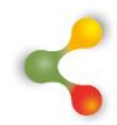

\section{REVUE HYBRIDE DE L'ÉDUCATION}

l'apprenant.e à reconnaitre par lui.elle-même la valeur relative des propositions scientifiques programmées.

Cette exigence que l'apprenant.e atteigne la conviction par lui.ellemême est essentielle. Cela ne veut pas dire qu'il fasse par lui.elle-même tout le travail de construction, mais que l'adhésion soit ultimement, au bout du processus, au moins partiellement délibérée et que la crédibilité relative soit volontairement accordée par l'intelligence même de l'apprenant.e. Évidemment, on peut souhaiter mieux que ce minimum, et même que l'intelligence se mette en marche bien plus intensivement, interactivement et résolument dans le processus de reconnaissance de la valeur des propositions programmées ou encore, qu'elle assume l'essentiel de la responsabilité dans l'exploration de leurs crédibilités relatives respectives (souvent obtenue par des études comparatives plus ou moins systématiquement menées).

Mais voilà : les élèves n'ont pas toujours choisi d'étudier les sciences. Alors que commencer par de petits pas suffira pour certains, même si nos ambitions pour eux sont évidemment plus grandes sur le long terme, un minimum de participation intellectuelle des élèves dans la reconnaissance de la valeur relative des propositions scientifiques permettra au moins de sécuriser que l'enseignement ne soit pas que persuasif. (tableau 2).

On reconnaît facilement les initiatives d'enseignement convaincant

\section{Tableau 2 : Caractérisation des interventions convaincantes}

\begin{tabular}{cc}
\hline Intervention & Buts recherchés \\
\hline $\begin{array}{c}\text { Les enseignant.e.s et les élèves perçoivent } \\
\text { les erreurs non pas comme des fautes à } \\
\text { corriger, mais comme des ressources pour } \\
\text { mieux apprendre (Astolfi, 1997). }\end{array}$ & $\begin{array}{c}\text { Éprouver la valeur des } \\
\text { propositions relativement à } \\
\text { des propositions } \\
\text { personnelles. }\end{array}$ \\
$\begin{array}{c}\text { On s'intéresse systématiquement aux } \\
\text { conceptions initiales des élèves (Duit et }\end{array}$ & $\begin{array}{c}\text { Éprouver la valeur des } \\
\text { propositions relativement à } \\
\text { Treagust, 2003) et on tente de les faire } \\
\text { évoluer par des dynamiques de réfutation, de } \\
\text { comparaisons, etc. }\end{array}$ \\
$\begin{array}{c}\text { On est attentif à la valeur ajoutée des } \\
\text { contenus scientifiques et on en privilégie }\end{array}$ \\
$\begin{array}{c}\text { certains plutôt que d'autres, en fonction de } \\
\text { cette valeur (Potvin, 2018). }\end{array}$ & $\begin{array}{c}\text { S'assurer que les } \\
\text { apprentissages à haute valeur } \\
\text { ajoutée soient sécurisés. }\end{array}$ \\
$\begin{array}{c}\text { On insiste sur les découvertes historiques, } \\
\text { mais surtout sur les contextes de rivalité } \\
\text { théorique qui se sont trouvés à leur origine } \\
\text { (Thouin, 2004) et dans lesquels ces }\end{array}$ & $\begin{array}{c}\text { Éprouver la valeur des } \\
\text { propositions relativement à }\end{array}$ \\
\hline
\end{tabular}




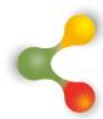

\section{REVUE HYBRIDE DE L'ÉDUCATION}

\begin{tabular}{|c|c|}
\hline $\begin{array}{l}\text { découvertes se sont avérées plus fertiles que } \\
\text { celles qui les précédaient. }\end{array}$ & $\begin{array}{l}\text { des propositions du passé } \\
\text { historique. }\end{array}$ \\
\hline $\begin{array}{c}\text { On reconnaît le caractère délibératif de } \\
\text { l'attribution des crédibilités scientifiques et on } \\
\text { en vient à simuler fidèlement des débats selon } \\
\text { cette façon de faire (Bell, 2004). On } \\
\text { encourage aussi les apprenants à mettre en } \\
\text { doute les propositions scientifiques et à } \\
\text { imaginer (et développer ?) des arguments, } \\
\text { des actions ou des dispositifs qui pourraient } \\
\text { tenter de les réfuter. }\end{array}$ & $\begin{array}{l}\text { Comprendre, expérimenter et } \\
\text { éprouver les mécanismes } \\
\text { d'attribution des crédibilités } \\
\text { scientifiques. Favoriser la } \\
\text { participation des élèves à ces } \\
\text { mécanismes. }\end{array}$ \\
\hline $\begin{array}{l}\text { On fait vivre des démarches d'investigation } \\
\text { scientifique aux élèves et on leur accorde } \\
\text { ainsi la possibilité de tester de manière } \\
\text { relativement autonome toutes sortes } \\
\text { d'hypothèses et d'avenues alternatives } \\
\text { d'exploration systématique des phénomènes } \\
\text { (Hasni, Belletête et Potvin, 2018), si possible } \\
\text { jusqu'à la présentation à des pairs de la } \\
\text { démarche retenue et des résultats avec } \\
\text { comme objectif de les convaincre sur cette } \\
\text { base. }\end{array}$ & $\begin{array}{l}\text { Comprendre, expérimenter et } \\
\text { éprouver les mécanismes } \\
\text { d'attribution des crédibilités } \\
\text { scientifiques. Favoriser la } \\
\text { participation des élèves à ces } \\
\text { mécanismes. }\end{array}$ \\
\hline $\begin{array}{l}\text { On s'interroge explicitement sur les raisons } \\
\text { pour lesquelles on résiste parfois à croire } \\
\text { certaines choses (psychologie); comment on } \\
\text { apprend mieux (métacognition) ou ce que sont } \\
\text { les critères qui font qu'une connaissance } \\
\text { particulière puisse être considérée meilleure } \\
\text { qu'une autre (épistémologie). }\end{array}$ & $\begin{array}{c}\text { Comprendre, expérimenter et } \\
\text { éprouver les contingences et } \\
\text { obstacles (de toutes sortes) à } \\
\text { l'attribution des crédibilités } \\
\text { scientifiques. }\end{array}$ \\
\hline $\begin{array}{l}\text { On s'efforce de susciter le besoin pour les } \\
\text { connaissances scientifiques à faire apprendre. }\end{array}$ & $\begin{array}{l}\text { Capitaliser sur la valeur } \\
\text { explicative, prédictive ou } \\
\text { exploitationelle des } \\
\text { propositions à faire } \\
\text { apprendre. }\end{array}$ \\
\hline
\end{tabular}

On reconnaît ici que plusieurs propositions pédagogiques souvent présentées comme innovantes ou souhaitables en sciences, comme les démarches d'investigation, pourraient être qualifiées de " convaincantes ». C'est peut-être parce que notre dichotomie s'appuie sur des valeurs ou des caractéristiques qu'on retrouve souvent dans ces propositions pédagogiques «à la mode ». Peut-être aussi s'agit-il simplement d'une parenté qui tient au fait que toutes ces activités considérées comme sophistiquées sont aisément reconnues, consciemment ou non, par les chercheur.e.s en éducation comme étant plus conformes à l'activité scientifique. Toutefois, si elles ont très souvent quelque chose de la " conviction", elles ne sont pas nécessairement centrées sur elle et pourraient entretenir d'autres buts. Elles ne mériteraient alors certainement pas toutes d'être réduites à cette étiquette. 


\section{$\&$}

\section{REVUE HYBRIDE DE L'ÉDUCATION}

Par contre, le concept d'enseignement convaincant pourrait certainement participer à la qualification qu'on fait de ces initiatives pédagogiques, en tant qu'adjectif plutôt que référent, et peut-être plus facilement encore être simplement opposées aux pédagogies "persuasives ». Notre proposition de dichotomie pourrait donc rester utile dans certains contextes de recherche et de formation, mais elle pourrait surtout donner des pistes à suivre pour une amélioration du caractère scientifique des interventions. Les questions auxquelles on cherche une réponse positive sont alors :

l'activité éducative prévue fournit-elle des informations et des expériences qui minimalement permettent

- d'éprouver, non pas uniquement de prouver, des propositions conceptuelles ou méthodologiques qui divergent des contenus des programmes, en plus de celles qui y sont inscrites?

- de réfléchir à la valeur objective des propositions scientifiques en fonction de normes épistémiques ? De réactions personnelles courantes (biais psychologiques, réflexions métacognitives) ? Ou d'autres contraintes liées à la valeur des propositions ?

- aux apprenant.e.s d'attribuer par eux-mêmes les crédibilités relatives des propositions scientifiques considérées?

À la fin de cette description, reconnaissons encore une fois que l'enseignement persuasif ne peut pas et ne doit pas être vu comme indésirable. II est, dans la réalité, nécessaire, mais lorsqu'un peu ou beaucoup d'espace moral ou temporel se dégage, l'idéal de l'enseignement convaincant pourrait être perçu comme une piste de développement didactique souhaitable, parce que claire et susceptible d'améliorer la scientificité et donc la qualité des interventions éducatives en sciences. En ce sens la proposition est fonctionnelle non seulement pour le chercheur, mais aussi pour le praticien. Reconnaissons tout de même que dans la réalité, le persuasif et le convaincant s'inscrivent en tension bien que l'on soit souvent contraint de choisir. Au moins, faisons-le consciemment.

\section{Apports et prospectives}

Notre proposition vise essentiellement à favoriser un étiquetage plus fonctionnel des interventions éducatives utilisées en sciences. Un des objectifs poursuivis est de soutenir, par un cadrage simple et dichotomique, mais évocateur de la valeur, des études expérimentales et quasi expérimentales comparatives qui testent des interventions en leur proposant une option que nous croyons intéressante et basée sur la nature et les propriétés exclusives à l'activité scientifique. II est envisagé qu'une meilleure identification ou une plus précise description des traitements 


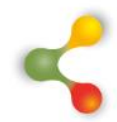

\section{REVUE HYBRIDE DE L'ÉDUCATION}

comparés qui pourrait découler de notre proposition permette de mieux apprécier la nature de la différence entre les groupes, de mieux cerner la variable indépendante, et donc, la cause précise des effets éventuellement enregistrés. Puisque la crédibilité des recherches en éducation est toujours à établir auprès des professionnels consommateurs de recherche, nous croyons que notre proposition est une contribution.

On pourrait aussi ambitionner que la formation des enseignant.e.s, initiale comme continue, puisse éviter certaines dérives en y référant explicitement, au lieu d'utiliser les dichotomies floues, relevant de l'angélisme, infantilisantes et blessantes. La formation offre un cadre intéressant aux éducateur.rice.s souhaitant que leur enseignement promeuve véritablement le développement de l'esprit scientifique. Même si la commande sociale et parfois même, leurs programmes, les entraînent vers un enseignement persuasif, plusieurs enseignant.e.s avaient déjà ressenti implicitement que «l'enseignement des résultats de la science n'est jamais un enseignement scientifique " (Bachelard, 1967) et ont souhaité aller plus loin. Plus souvent, on leur a proposé des horizons aux contours flous, sans vraiment leur offrir de critères de qualification clairs pour les interventions envisagées. On les a aussi invités à répétition à "révolutionner leur pratique », ce qu'ils ou qu'elles ne feront évidemment pas. Mais peut-être avec des images claires et avec la possibilité de transformer progressivement leurs actions parviendront-ils à faire comme les professionnel.le.s de la santé et faire évoluer leur profession.

Ici, la clé proposée n'est pas que pédagogique ; elle est d'ordre didactique, et donc probablement davantage propre à interpeller les enseignant.e.s de science. Par prudence, nous avons refusé de l'étendre aux autres disciplines scolaires, qui parfois, comme en langues, enseignent des conventions humaines plus que des phénomènes naturels. Peut-être pourrait-on pousser l'audace jusqu'à suggérer qu'elle puisse aussi servir dans l'enseignement des sciences humaines, dont la sensibilité aux réalités historiques et sociales cette fois, est aussi forte qu'en sciences.

Pour l'instant, la valeur de la dichotomie proposée n'est qu'une hypothèse, mais nous croyons qu'elle pourrait aussi faire l'objet d'une programmation de recherche exhaustive qui se mettrait en projet d'en tester les effets sur toutes sortes de variables perceptuelles et académiques. Même si de nombreuses idées d'applications concrètes du tandem persuasif-convaincant (pour des contenus et compétences spécifiques) nous viennent à l'esprit et nous semblent nécessaires pour mieux illustrer ce que nous voulons dire, il faudra éventuellement remettre cela à une prochaine contribution.

Finalement, nous entrevoyons qu'il soit possible et vraisemblablement nécessaire pour des besoins de cohérence d'importer le binôme dans la formation des enseignant.e.s non seulement pour ses objets, mais aussi pour la manière dont on choisira de l'opérer. La formation 


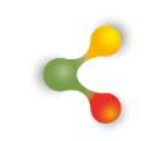

\section{REVUE HYBRIDE DE L'ÉDUCATION}

des enseignant.e.s est une mise en abîme qui exige qu'on fasse ce qu'on prêche. Il serait en effet assez paradoxal et regrettable que des formations à l'enseignement convaincant soient menées en mode persuasif. Du grain à moudre pour les formateur.trice.s responsables. 


\section{REVUE HYBRIDE DE L'ÉDUCATION}

\section{Références}

Albe, V. (2005). Un jeu de rôle sur une controverse socio-scientifique actuelle : Une stratégie pour favoriser la problématisation? Aster, (40), 67-94. DOI:10.4267/2042/8856

Astolfi, J.-P. (1997). L'erreur, un outil pour enseigner. ESF Éditeur.

Bachelard, G. (1963). Le nouvel esprit scientifique. Presses universitaires de France.

Bachelard, G. (1967). La formation de l'esprit scientifique. Librairie Vrin.

Bell, P. (2004). Promoting students' argument construction and collaborative debate in the science classroom. Dans M.C. Linn, E.A. Davis et P. Bell (dir.). Internet environments for science education ( $\mathrm{p}$. 143-172). Lawrence Erlbaum Associates Publishers.

Boudreau, M. et Beaudoin, I. (2015). L'album documentaire, un incontournable pour favoriser l'entrée dans l'écrit. Revue de recherches en littératie médiatique multimidale, 2(Octobre), 1-31. DOI:10.7202/1047308ar

Brod, G., Hasselhorn, M. et Bunge, S. A. (2018). When generating a prediction boosts learning: The element of surprise. Learning and Instruction, 55, 22-31. DOI:10.1016/j.learninstruc.2018.01.013

Duit, R. et Treagust, D. (2003). Conceptual change - A powerful framework for improving science teaching and learning. International Journal of Science Education, 25(6), 671-688. DOI:10.1080/09500690305016

Engelmann, S., Carnine, D. et Steely, D. G. (1991). Making Connections in Mathematics. Journal of Learning Disabilities, 24(5), 292-303. DOI:10.1177/002221949102400506

Feynman, R. P., Cox, B. et Ma, Y.-Y. (2015). The quotable Feynman. Princeton University Press.

Fourez, G. (2002). Les sciences dans l'enseignement secondaire. Didaskalia, 21, 107-122. doi:10.4267/2042/25107

Geis, G. L. (1984). Comparing Instructional Methods: Some Basic Research Problems. Canadian Journal of Higher Education, 14(2), 91-98.

Hasni, A. et Potvin, P. (2015). L'intérêt pour les sciences et la technologie à l'école : Résultats d'une enquête auprès d'élèves du primaire et du secondaire au Québec. Chaire de recherche sur l'intérêt des jeunes à l'égard des sciences et de la technologie (CRIJEST). http://crijest.org/sites/crijest.org/files/Hasni-Potvin-RapportCRIJEST-2015-VF.pdf

Hasni, A., Belletête, V. et Potvin, P. (2018). Les démarches d'investigation scientifique. Un outil de réflexion sur les pratiques de classe. Centre 


\section{REVUE HYBRIDE DE L'ÉDUCATION}

de recherche sur l'enseignement et l'apprentissage des sciences / Chaire de recherche sur l'intérêt des jeunes à l'égard des sciences et de la technologie.

Kirschner, P. A., Sweller, J. et Clark, R. E. (2006). Why Minimal Guidance During Instruction Does Not Work: An Analysis of the Failure of Constructivist, Discovery, Problem-Based, Experiential, and InquiryBased Teaching. Educational Psychologist, 41(2), 75-86. DOI:10.1207/s15326985ep4102_1

Kuhn, T. S. (1962). La structure des révolutions scientifiques. ChampsFlammarion.

Le Petit Robert. (2020). Dictionnaire Le Petit Robert. Robert Ed.

Legendre, R. (1983). L'éducation totale. Fernand-Nathan.

Legendre, R. (1995). Entre l'angoisse et le rêve. Guérin/Eska.

Li, J. et Klahr, D. (2006). The psychology of scientific thinking: Implications for science teaching and learning. Dans J. Rhoton et P. Shane (dir.) Teaching Science in the 21st Century. National Science Teachers Association and National Science Education Leadership Association: NSTA Press.

Lilly, E. L. (2012). Assigned Positions for In-Class Debates Influence Student Opinions. International Journal of Teaching and Learning in Higher Education, 24(1), 1-5.

Popper, K. R. (1995). La logique de la découverte scientifique. Éditions Payot.

Popper, K. R., De Launay, M.-I. et De Launay, M. B. (1985). Conjectures et réfutations: la croissance du savoir scientifique. Éditions Payot.

Potvin, P. et Hasni, A. (2014). Interest, motivation and attitude towards science and technology at K-12 levels: a systematic review of 12 years of educational research. Studies in science education, 50(1), 85-129. doi: 10.1080/03057267.2014.881626

Potvin, P. (2018). Faire apprendre les sciences et la technologie au secondaire. Épistémologie, Didactique, Sciences cognitives et Neurosciences au service de l'enseignant. Presses de l'Université Laval.

Reigosa, C. et Jiménez-Aleixandre, M. P. (2007). Scaffolded Problemsolving in the Physics and Chemistry Laboratory: Difficulties hindering students' assumption of responsibility. International Journal of Science Education, 29(3), 307-329. DOI:10.1080/09500690600702454

Schraw, G., Crippen, K. J. et Hartley, K. (2006). Promoting Self-Regulation in Science Education: Metacognition as Part of a Broader 


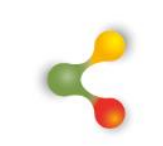

\section{REVUE HYBRIDE DE L’ÉDUCATION}

Perspective on Learning. Research in science education, 36(1), 111139. DOI:10.1007/s11165-005-3917-8

Schroeder, C. M., Scott, T. P., Tolson, H., Huang, T.-Y. et Yi-Hsuang, L. (2007). A meta-analysis of national research: effets of teaching strategies on student achievement in science in the United States. Journal of Research in Science Teaching, 44(10), 1436-1460. https://doi.org/10.1002/tea.20212

Thouin, M. (2004). Explorer l'histoire des sciences et des techniques. Activités, exercices et problèmes. Multimondes. 